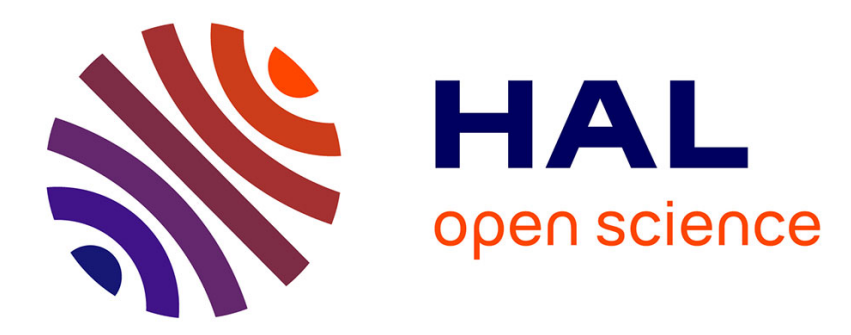

\title{
Épizooties et efficacité des processus de décision : un exemple en apiculture \\ Jean-Philippe Terreaux
}

\section{To cite this version:}

Jean-Philippe Terreaux. Épizooties et efficacité des processus de décision: un exemple en apiculture. 2017, 32 (2), pp.160-197. hal-02399496

\section{HAL Id: hal-02399496 \\ https://hal.science/hal-02399496}

Submitted on 9 Dec 2019

HAL is a multi-disciplinary open access archive for the deposit and dissemination of scientific research documents, whether they are published or not. The documents may come from teaching and research institutions in France or abroad, or from public or private research centers.
L'archive ouverte pluridisciplinaire HAL, est destinée au dépôt et à la diffusion de documents scientifiques de niveau recherche, publiés ou non, émanant des établissements d'enseignement et de recherche français ou étrangers, des laboratoires publics ou privés. 


\title{
EPIZOOTIES ET EFFICACITÉ DES PROCESSUS DE DÉCISION : UN EXEMPLE EN APICULTURE
}

\author{
Jean-Philippe Terreaux
}

Revue française d'économie | «Revue française d'économie »

2017/2 Vol. XXXII | pages 160 à 197

ISSN 0769-0479

Article disponible en ligne à l'adresse :

https://www.cairn.info/revue-francaise-d-economie-2017-2-page-160.htm

\section{Pour citer cet article :}

Jean-Philippe Terreaux, «Epizooties et efficacité des processus de décision : un exemple en apiculture », Revue française d'économie 2017/2 (Vol. XXXII), p. 160-197.

DOI $10.3917 /$ rfe.172.0160

Distribution électronique Cairn.info pour Revue française d'économie.

(C) Revue française d'économie. Tous droits réservés pour tous pays.

La reproduction ou représentation de cet article, notamment par photocopie, n'est autorisée que dans les limites des conditions générales d'utilisation du site ou, le cas échéant, des conditions générales de la licence souscrite par votre établissement. Toute autre reproduction ou représentation, en tout ou partie, sous quelque forme et de quelque manière que ce soit, est interdite sauf accord préalable et écrit de l'éditeur, en dehors des cas prévus par la législation en vigueur en France. Il est précisé que son stockage dans une base de données est également interdit. 


\section{Jean-Philippe TERREAUX}

\section{Epizooties et efficacité des processus de décision : un exemple en apiculture}

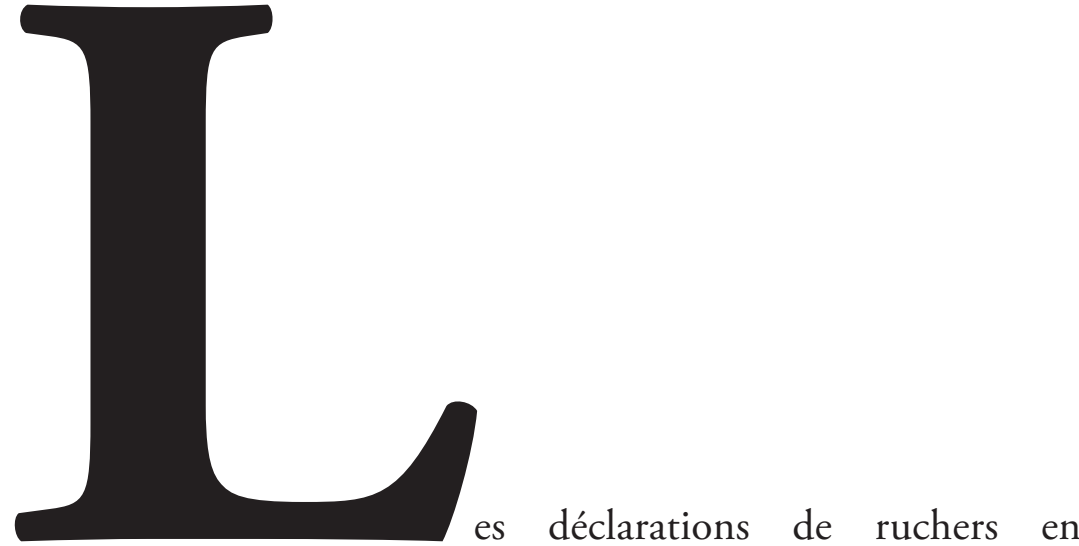

Aquitaine, font état de 1507 apiculteurs en 2014, certains ne possédant qu'une seule ruche alors que d'autres en détiennent plus d'un millier. Cette hétérogénéité est encore accrue si l'on tient compte des situations financières, économiques, sociales 
etc., de ces agents. Afin de lutter contre différentes pathologies des abeilles, la loi permet aujourd'hui aux apiculteurs de se regrouper au sein d'organismes reconnus par l'État (organismes à vocation sanitaire - OVS) et de prendre ensemble des décisions engageant l'avenir de leur propre activité et celui de l'ensemble des apiculteurs, et ayant indirectement des répercussions concernant toute la société. Les modalités de prise de décision au sein de ces organismes ont pourtant peu été l'objet de réflexions, or nous montrons ici qu'il s'agit d'un point essentiel à partir de l'exemple de la lutte contre la loque américaine.

Lobjectif de cet article est de montrer que face à une pathologie importante, à diffusion " horizontale " c'est-à-dire d'une colonie ${ }^{1}$ à ses voisines, pour laquelle la complexité de l'organisation actuelle conduit à une situation peu efficiente, il serait utile de revenir à un mode de décision démocratique simple, adapté et efficace. En effet, différents moyens de lutte existent, mais nécessitent une coordination plus ou moins forte entre les apiculteurs, et surtout ces moyens n'ont pas les mêmes impacts économiques selon la taille des ruchers.

Plus généralement, chaque apiculteur lutte contre les maladies de son rucher, ce qui modifie le risque de diffusion de la maladie aux ruchers voisins. Il s'agit ainsi d'une externalité positive et, sans action concertée entre les apiculteurs, l'effort global restera globalement sous-optimal ; ce problème est commun à la production de nombreux biens publics, dont le contrôle des épidémies ou des épizooties est souvent présenté comme un archétype. C'est une des raisons de la mise en place des OVS. Si l'établissement de cette structure relève plutôt de la théorie de l' "implementation " (ou de la "mise en œuvre ", à savoir la recherche des modalités de coordination ou d'institution les plus à même d'atteindre un objectif donné ; voir Kakhbod [2013]), son fonctionnement est plutôt du ressort du " mechanism design " (" conception de mécanisme ", c'est à dire, une fois cette structure en place, définir une méthode permettant d'atteindre un objectif acceptable ; voir Baliga et Maskin [2003]). 
En outre, pourrait se poser un problème d'asymétrie d'information, lorsque l'apiculteur ne déclare pas au coordinateur la présence du pathogène dans ses colonies. En principe, la déclaration de la présence de la loque américaine est obligatoire. Mais deux problèmes se posent : d'une part une difficulté à sa détection, le plus souvent due au manque de connaissances chez les apiculteurs, en particulier chez les amateurs, et d'autre part la déclaration elle-même, problème qui peut être commun à d'autres pathologies, en apiculture ou pour d'autres systèmes de production. Cette dernière cause de l'asymétrie d'information est particulièrement difficile à résoudre, lorsque les apiculteurs ne sont pas indemnisés pour les coûts directs et indirects des actions entreprises, actions dont bénéficie l'ensemble de la société.

Dans la suite de ce document, nous développons un modèle (une représentation simplifiée de la réalité) qualitatif qui permet de mettre en évidence que l'hétérogénéité des apiculteurs, mesurée de la manière la plus simple par la taille des ruchers, et la diversité des modalités de diffusion des pathologies obligent à prendre des décisions coordonnées au sein d'un collectif, l'OVS, qui trouve avantage à regrouper l'ensemble des apiculteurs; ces décisions pourront se traduire par des contraintes sur l'effort minimal de lutte de chacun, dans l'intérêt de chaque apiculteur, de l'ensemble des apiculteurs, et indirectement de la collectivité qui bénéficie en particulier de la présence des pollinisateurs. Ces efforts sont liés directement au mode de décision mis en place dans l'OVS (par exemple un vote à la majorité simple versus un vote à la majorité qualifiée, c'est-à-dire avec pondération de chaque vote). L'avenir de l'apiculture et de ses aménités pour la collectivité dépend ainsi tout autant de l'organisation du fonctionnement de l'OVS que des différents paramètres de diffusion des pathogènes, ou que des autres causes de la baisse des effectifs des abeilles qui sont mises en avant (pesticides, frelon asiatique etc.).

Le modèle représente l'évolution des colonies actives dans les ruchers de la région considérée, l'Aquitaine ${ }^{2}$, avant sa fusion avec deux autres régions pour fonder la " Nouvelle Aquitaine ». 
Ce sujet est d'autant plus important que le regroupement des régions va conduire à définir les modalités de décision dans la nouvelle structure qui sera mise en place en conséquence. Nous travaillons sur l'exemple de la loque américaine (voir Genersch [2010] ; Mill et al. [2014] ; Datta et al. [2015]), une pathologie particulièrement virulente.

Dans la première section nous décrivons, sur le plan quantitatif, l'hétérogénéité de la taille des ruchers. Ensuite, nous établissons un modèle qualitatif, le plus simple, nous permettant d'aboutir à notre conclusion. Puis nous présentons les résultats obtenus. Nous donnons quelques commentaires en conclusion.

\section{Le modèle}

\section{Hétérogénéité de la taille des ruchers : la vulnérabilité}

De manière à préciser quantitativement l'ampleur de l'hétérogénéité des ruchers, nous traçons la courbe de Lorenz de leur taille (Lorenz [1905]), à partir des déclarations faites en Aquitaine (données de 2014).

Soit $k$ un numéro d'ordre attribué à chaque apiculteur ( $1 \leq k \leq n, n$ étant le nombre total d'apiculteurs dans la région), numéro attribué en ordre croissant en fonction du nombre de ruches. Soit $F(k)$ le nombre total de ruches, appartenant à des apiculteurs dont le numéro d'ordre est inférieur à $k$. Pour l'Aquitaine en 2014, à partir des déclarations de ruchers par les apiculteurs, la courbe $F(k)$, dite courbe de Lorenz, est représentée en figure $\mathrm{n}^{\circ} 1$. Sa forte convexité illustre la grande hétérogénéité des apiculteurs vis-à-vis de la taille des ruchers. Le grand segment de droite correspondrait à une distribution égale du nombre de ruches par apiculteur. 
Figure 1

\section{Taille des ruchers des apiculteurs d'Aquitaine : courbe de Lorenz et approximation de cette courbe par trois segments de droite}

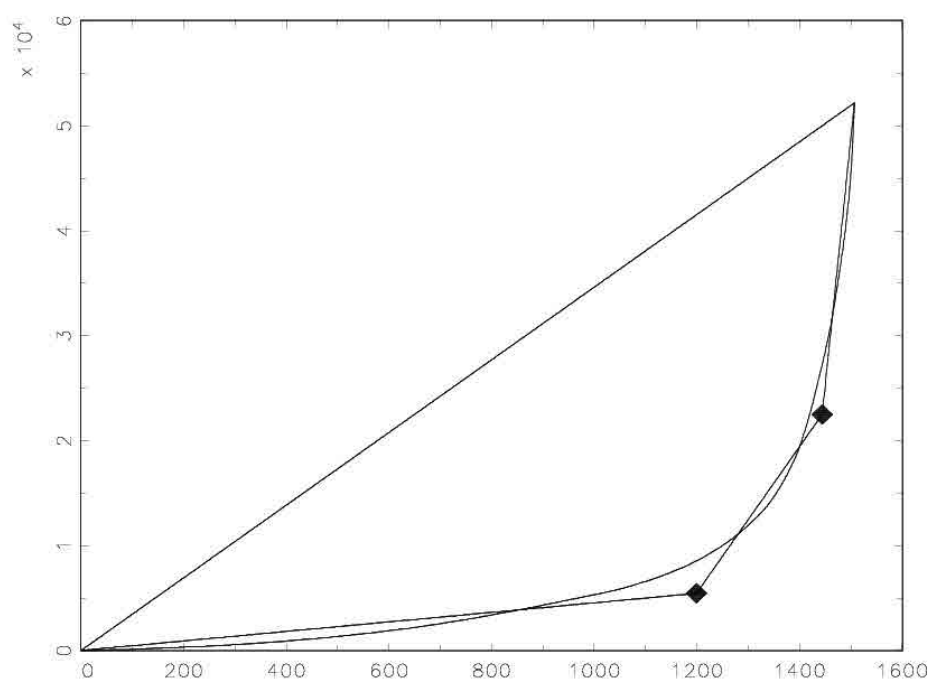

Afin de simplifier la représentation du nombre de ruches par apiculteur, nous approchons cette courbe par trois segments de droite, dont on peut calculer les abscisses et ordonnées des deux points d'intersection : les quatre variables (abscisses et ordonnées des deux points intermédiaires) peuvent être optimisées pour minimiser la surface séparant la courbe des trois segments de droite. La figure $\mathrm{n}^{\circ} 1$ nous montre le résultat de cette optimisation.

Finalement, il est possible de considérer, sans trop trahir la réalité, pour ce qui concerne notre modèle, trois types d'apiculteurs : grands, moyens et petits, dont les pentes des segments de droite nous donnent le nombre moyen de colonies, et les abscisses des points d'intersection entre ces segments le nombre d'apiculteurs de chaque catégorie (données de 2014). 
Tableau 1

\section{Représentation des ruchers d'Aquitaine dans le modèle utilisé}

\begin{tabular}{|l|c|c|c|c|c|}
\hline & $\begin{array}{l}\text { Nombre } \\
\text { moyen de } \\
\text { colonies }\end{array}$ & $\begin{array}{l}\text { Nombre } \\
\text { d'apiculteurs }\end{array}$ & $\begin{array}{l}\text { Nombre } \\
\text { minimal de } \\
\text { colonies }\end{array}$ & $\begin{array}{l}\text { Nombre } \\
\text { maximal de } \\
\text { colonies }\end{array}$ & $\begin{array}{l}\text { Nombre } \\
\text { total de } \\
\text { colonies }\end{array}$ \\
\hline Petits & 4,54 & 1199 & 1 & 25 & 5440 \\
\hline Moyens & 69,59 & 245 & 26 & 200 & 17060 \\
\hline Grands & 471,75 & 63 & 201 & - & 29699 \\
\hline $\begin{array}{l}\text { Toutes } \\
\text { catégories }\end{array}$ & 34,64 & 1507 & 1 & - & 52199 \\
\hline
\end{tabular}

C'est à partir de cette représentation que nous construisons dans les sections suivantes notre modèle de diffusion de la pathologie. Ce dernier est fait dans un but précis, et ses résultats ne peuvent que très précautionneusement être étendus à d'autres objectifs. Nous ne tenons pas compte explicitement dans cette section de l'éclatement fréquent des grands ruchers en "sous-ruchers" de moindre taille ; mais, pour la pathologie concernée par ce travail, il est clair que la mobilité de l'apiculteur et de ses ruches entre les différents sous-ruchers peut entrầner une diffusion de la pathologie différente de celle observée d'un rucher à l'autre (voir sur ce point, par exemple, Datta et al. [2015]). D'où l'intérêt de conserver une distinction entre les grands ruchers et les ruchers moyens, auxquels nous aurions pu sinon rattacher les sous-ruchers.

\section{Représentation des utilités}

Nous définissons l'utilité des différents apiculteurs, de l'ensemble des apiculteurs et de la société. Nous ne tenons pas compte de l'aversion au risque dans ce modèle qui est déterministe.

\section{Utilité de chaque apiculteur}

Dans un objectif de simplification, pour une année considérée isolément, l'utilité $u(i)$ de chaque apiculteur $i$ peut être calculée 
comme une somme pondérée de la production de miel l'année considérée et du nombre de colonies actives, sources d'aménités (il s'agit aussi d'un gage de production de miel futur, mais cette production à venir est prise en compte dans le cadre de la somme actualisée des utilités) moins le montant des impôts, taxes et cotisations diverses (mutualité sociale agricole par exemple). On aura ainsi pour chaque année :

$$
u(i)=p(i) \cdot m(i)+v(i) \cdot N(i)-\operatorname{tax}(i)
$$

avec $p(i)$ le prix du miel vendu par l'apiculteur $i, m(i)$ la production de miel de l'apiculteur $i, v(i)$ l'utilité pour $i$ liée à la présence même d'une colonie, incluant les autres biens (par exemple vente de cire, de reines, d'essaims) et services (par exemple pour la pollinisation) rémunérés. $N(i)$ est le nombre de colonies de l'apiculteur $i$; $m(i)$ n'est pas proportionnel à $N(i)$, les ruches en voie de rémission de la pathologie produisant moins de miel (voir annexe $\mathrm{n}^{\circ} 1$ ). On notera que $p(i)$ et $v(i)$ peuvent différer selon les apiculteurs et les modalités de commercialisation et de rémunération disponibles ; $\operatorname{tax}(i)$ est le montant des impôts et cotisations.

\section{Utilité collective des apiculteurs}

L'utilité collective des apiculteurs est notée $u(A)$. Elle peut se définir comme la somme pondérée, avec des facteurs $q_{i}$, des utilités de chaque apiculteur pour l'année considérée.

$$
\mathrm{u}(\mathrm{A})=\sum_{i=1}^{n} q_{i} u(i)
$$

Dans la suite de l'article, on prendra $q_{i}=1$, c'est-à-dire que les contributions de chaque apiculteur à l'utilité collective ne sont pas différenciées. 


\section{Utilité de l'État}

On considère que l'utilité de l'État issue de l'apiculture provient de l'utilité des apiculteurs et de celle des autres membres de la société.

De manière pragmatique, on calculera ici l'utilité de l'État comme la somme de la valeur de la pollinisation, des impôts perçus sur le revenu, des cotisations MSA (qui d'ailleurs en deçà de 200 ruches n'ouvrent aucun droit), ainsi que d'un pourcentage des utilités des apiculteurs afin de représenter l'utilité économique, sociale et environnementale de cette production (utilité propre des apiculteurs et effet de sillage sur la société).

\section{Prise en compte du futur}

Afin de tenir compte des utilités des différentes parties prenantes au fil du temps, et notamment l'intérêt économique de mettre en place des mesures coûteuses à court terme mais augmentant l'utilité à long terme, on actualise les différentes utilités avec un taux $\tau(t)$, $t$ étant l'indice de l'année, en suivant les recommandations du CGP (2005), à savoir un taux marginal d'actualisation de $4 \%$ les trente premières années puis de $2 \%$ (voir, sur les possibles problèmes de cohérence induits par des taux décroissants, Terreaux [2008]). Cela nous permet aussi de comparer numériquement les utilités obtenues pour différentes actions et de ne pas avoir à comparer visuellement différents graphiques.

\section{Efforts des apiculteurs et effets externes}

Supposons que chaque apiculteur $i$ fasse un effort de lutte contre une pathologie, effort représenté par son coût $e_{i} \geq 0$, et que le résultat des efforts combinés soit une modification de l'utilité $u(i)$ de l'apiculteur, modification définie par un facteur multiplicatif $w\left(\sum_{i} e_{i}\right)$, avec $w^{\prime}>0$ et $w^{\prime \prime}<0$. Alors l'effort de chaque apiculteur se déduit de la solution : 
168 Jean-Philippe Terreaux

$$
\max _{e_{i}} w\left(\sum_{i} e_{i}\right) \cdot u_{i}-e_{i}
$$

Si les actions de lutte sont menées par les apiculteurs indépendamment les uns des autres, les conditions nécessaires d'optimalité vont conduire à une solution en termes de coût $e_{i}^{0}$ satisfaisant (équation (1)) :

$$
\left\{\begin{array}{c}
W^{\prime}\left(\sum_{j \neq i} e_{j}+e_{i}^{0}\right) \cdot u_{i}-1=0 \text { si } e_{i}^{0}>0 \\
W^{\prime}\left(\sum_{j \neq i} e_{j}\right) \cdot u_{i}-1<0 \text { si } e_{i}^{0}=0
\end{array}\right.
$$

Supposons qu'un groupe $I$ d'apiculteurs puisse mener des actions coordonnées d'un coût global $\sum_{i \in I} \hat{e}_{i}$. L'effort optimal de ce groupe satisfera les conditions nécessaires d'optimalité suivantes :

$$
\left\{\begin{array}{c}
w^{\prime}\left(\sum_{j \notin I} \hat{e}_{j}+\sum_{i \in I} \hat{e}_{i}\right) \cdot \sum_{i \in I} u_{i}-1=0 \quad \text { si } \sum_{i \in I} \hat{e}_{i}^{I}>0 \\
w^{\prime}\left(\sum_{j \notin I} \hat{e}_{j}\right) \cdot \sum_{i \in I} u_{i}-1<0 \text { si } \sum_{i \in I} \hat{e}_{i}^{I}=0
\end{array}\right.
$$

Dans le cas où les efforts ne sont pas nuls, on remarquera que :

$$
W^{\prime}\left(\sum_{j \neq i} e_{j}+e_{i}^{0}\right)=\frac{1}{u_{i}}>\frac{1}{\sum_{i \in I} u_{i}}=W^{\prime}\left(\sum_{j \notin I} \hat{e}_{j}+\sum_{i \in I} \hat{e}_{i}^{I}\right)
$$


Comme $w^{\prime}<0$, on en déduit que :

$$
\sum_{j \neq i} e_{j}+e_{i}^{0}<\sum_{j \notin I} \hat{e}_{j}+\sum_{i \in I} \hat{e}_{i}^{I}
$$

C'est-à-dire que les efforts induits par l'existence du collectif $I$ sont plus importants que ceux qui sont menés lorsque ce collectif n'existe pas. Ils le seront d'autant plus que l'inégalité est importante dans l'inéquation précédente, autrement dit d'autant plus que le collectif $I$ est grand en termes d'efforts coalisés.

Il serait ainsi tentant pour les apiculteurs et pour la puissance publique de mettre en place le collectif le plus grand possible, noté $N$, c'est-à-dire regroupant tous les apiculteurs, permettant ainsi d'atteindre la solution de :

$$
\max _{e_{i}}\left(W\left(\sum_{i} \hat{e}_{i}\right)\right) \cdot\left(\sum_{i} u_{i}\right)-\sum_{i} \hat{e}_{i}
$$

qui correspond au maximum de variation nette d'utilité atteignable par l'ensemble des apiculteurs, notée $U$. Il faudrait pour cela mettre en place un système de calcul et de redistribution de ces variations nettes d'utilité vis-à-vis de la situation où les actions ne sont pas coordonnées, le montant de ces variations s'élevant à :

$$
\left[W\left(\sum_{i} \hat{e}_{i}^{N}\right)-W\left(\sum_{i} e_{i}^{0}\right)\right] \cdot\left(\sum_{i} u_{i}\right)-\left(\sum_{i} \hat{e}_{i}^{N}-\sum_{i} e_{i}^{0}\right)
$$

calculé de sorte que, en recevant une part $U_{\mathrm{i}}$ de $U$, avec $U=\sum_{\mathrm{i}} U_{\mathrm{i}}$, et en résolvant :

$$
\max _{e_{i}} W\left(\sum_{j \neq i} e_{j}+e_{i}\right) \cdot\left(u_{i}+U_{i}\right)-e_{i}
$$


l'apiculteur $i$ soit conduit à faire l'effort optimal de coût $\hat{e}_{i}^{N}$. Comme le calcul des $U_{\mathrm{i}}$ est très compliqué, il n'est pas envisageable de mettre en pratique une telle subvention individuelle.

Le risque est alors important de voir apparaître des " passagers clandestins ", à savoir des apiculteurs qui profitent des efforts des autres, sans faire un effort à la hauteur en retour. Ce risque est avéré lorsque pour un apiculteur la solution $e_{i}^{0}=0$ est optimale, c'est-à-dire, d'après l'équation (1), lorsque :

$$
u_{i}<\frac{1}{W^{\prime}\left(\sum_{j \neq i} e_{j}\right)}
$$

Autrement dit, tous les apiculteurs dont l'utilité est inférieure à une certaine valeur vont avoir un niveau d'effort nul, et seuls les apiculteurs pour lesquels l'enjeu est suffisamment important feront un effort de lutte.

On peut noter aussi que dans le cas de certaines pathologies particulièrement virulentes, le facteur multiplicatif de l'efficacité peut ne pas être fonction de la somme des efforts, représentée par $\sum_{i} e_{i}$, mais de l'effort minimum $\operatorname{Min}_{i} e_{i}$. Dans ce cas il suffit qu'un seul apiculteur ne fasse pas d'effort pour que l'augmentation d'utilité soit nulle. Chaque apiculteur résout alors

$$
\max _{e_{i}} w\left(\operatorname{Min}_{j} e_{j}\right) \cdot u_{i}-e_{i}
$$

de solution :

$$
\left\{\begin{array}{c}
w^{\prime}\left(e_{i}^{0}\right) \cdot u_{i}-1=0 \text { soit } w^{\prime}\left(e_{i}\right)=\frac{1}{u_{i}}, \text { si } e_{i}^{0}>0 \text { et avec } \forall j \neq i, e_{j}^{0} \geq e_{i}^{0}, \\
e_{i}^{0}=0 \text { sinon, quelle que soit la valeur de } w^{\prime}(0) \cdot u_{i}-1
\end{array}\right.
$$


La solution sera non nulle pour tous les $i$, s'il existe une solution non nulle satisfaisant les conditions nécessaires d'optimalité pour chacun des apiculteurs, ce qui nécessite que :

$$
\forall i, \exists e_{i}>0 / w^{\prime}\left(e_{i}\right) \cdot u_{i}-1>0,
$$

c'est-à-dire si :

$$
\forall i, \exists e_{i}>0 / u_{i}>\frac{1}{w^{\prime}\left(e_{i}\right)}
$$

Ce n'est pas garanti si certains apiculteurs ont des utilités trop faibles par rapport au coût de l'effort individuel. Si une coalition $I$ est mise en place, coalition qui impose à chacun de ses membres un effort minimal $e^{I}$, l'équation devient :

$$
\left\{\begin{array}{c}
\operatorname{Max}_{e_{i}} W\left(\operatorname{Min}_{j}\left(e_{j}\right)\right) \cdot u_{i}-e_{i} \\
\text { sous contrainte }: e_{i}>e^{I}, \forall i \in I
\end{array}\right.
$$

La solution sera non nulle si $\forall j \notin I, \exists e_{j}>0 / u_{j}>\frac{1}{w^{\prime}\left(e_{j}\right)}$

Autrement dit, cette coalition, pour conduire à un effort non nul, aura intérêt à intégrer tous les apiculteurs (ici, ceux dont les utilités sont les plus faibles) qui sinon ne feraient pas cet effort minimum. En résumé, pour arriver à l'efficacité globale souhaitée, la coalition devra imposer un niveau d'effort individuel $e^{I}$ à tous les apiculteurs qui naturellement ne feraient pas cet effort. Comme pour les autres apiculteurs ce niveau est déjà atteint ou dépassé, elle a intérêt par simplicité à imposer cette contrainte à chacun des apiculteurs sans distinction.

On a vu dans les deux cas, que l'efficacité des mesures soit fonction de la somme des efforts ou de l'effort minimum, qu'il est important de mettre en place un collectif le plus large 
possible, c'est-à-dire intégrant l'ensemble des apiculteurs et, afin d'éviter des comportements de passager clandestin ou des calculs très compliqués, d'imposer des règles d'effort à ce collectif. C'est la raison d'être et le rôle des OVS. Mais ces règles, on l'a également vu, n'auront pas le même intérêt, ni le même coût pour chaque apiculteur, comme cela sera illustré numériquement dans les sections suivantes. Aussi les modalités de décision à l'intérieur de ce collectif doivent-elles tenir compte de l'hétérogénéité des apiculteurs, et notamment de la taille de leur rucher, comme nous l'avons constaté sur un des cas précédents.

\section{Règle de décision - variable de commande}

Face à la diffusion d'un pathogène, différentes mesures peuvent être prises, visant par exemple la diminution de la probabilité de contamination d'une colonie à l'autre dans un même rucher, d'un rucher à l'autre, ou encore visant la diminution des effets sur les colonies infestées. Nous avons vu que les différences de taille entre les ruchers font que les décisions optimales pour certains apiculteurs ne le sont pas pour d'autres. Les intérêts de l'État (au sens de la puissance publique) peuvent aussi différer de ceux des apiculteurs. Enfin des effets de passagers clandestins peuvent être observés.

À cause de la complexité du contexte, il nous faut revenir aux méthodes fondamentales pour faire un choix social. Dans un cadre démocratique, Knight [1947] les résume en trois catégories : habitude, autorité ou consensus. L'habitude ou le statu quo, ou encore l'adaptation lente, n'est pas compatible avec l'apparition de nouvelles pathologies, de nouveaux problèmes, ni avec le changement global (changement des modes culturaux agricoles et des spéculations, changement dans le monde de l'apiculture, modification des objectifs, évolution des valeurs etc.).

La deuxième catégorie, l'autorité, qui ne pourrait être que celle de l'État, imposant ses vues et des mesures obligatoires aux apiculteurs, peut ne pas tout à fait correspondre à ce que l'on attend d'une démocratie (voir par exemple Terreaux 
[2014]) ; dans notre cadre, la divergence d'intérêt entre l'État et les apiculteurs deviendrait vite un obstacle à une adhésion des apiculteurs aux mesures imposées, et conduirait à la nécessité de mise en place de méthodes de contrôle économiquement et socialement coûteuses. Une autre méthode assez autoritaire peut se traduire par la mise en œuvre de taxes et de subventions fortement incitatives, mais avec d'autres problèmes d'ajustement des niveaux de prélèvement et de restitution, telles que celles que nous avons mises en évidence dans la section précédente, et finalement des difficultés d'efficacité globale, notamment à cause des coûts indirects induits.

Il reste le consensus. K.J. Arrow [1963] nous conduit à la même conclusion et détaille adroitement la notion de consensus à laquelle il rattache les mécanismes de vote et de marché. Mais, dans notre cas, ces derniers sont peu opérants puisque seuls le miel et quelques autres produits sont commercialisés, alors que toutes les aménités et externalités positives des activités des abeilles échappent aux marchés (voir par exemple Gallai et al. [2009]). En outre, on ne voit guère comment ces derniers pourraient résoudre le problème de la diffusion de pathogènes, au vu de la facilité d'importer du miel de pays étrangers qui ne sont pas nécessairement exposés aux mêmes pathologies.

Subsistent alors les mécanismes de vote, dans une acception étendue, à savoir de décision par des groupes plus ou moins larges des principales personnes concernées. Cette délégation par l'État de la décision est d'autant plus judicieuse que l'intérêt collectif n'est pas défini : Joseph Priestley [1768] suggérait bien d'essayer d'aboutir au "plus grand bonheur du plus grand nombre ", mais Arrow [1963] soulignait que même si l'on connaissait les intérêts individuels, on ne saurait comment les agréger dans une formulation acceptable. Plus généralement (voir Terreaux [2014]) peut-être s'agit-il ici d'assurer le développement ou tout au moins une certaine stabilité à l'apiculture et à l'agriculture, une certaine pérennité sociale et environnementale, une certaine continuité des structures.

Dans ce cadre, on comprend facilement comment on a pu aboutir au fil du temps à la situation actuelle, qui reste perfec- 
tible. Les apiculteurs doivent tous déclarer leur rucher à l'administration. Ils peuvent adhérer au Groupement de défense sanitaire apicole de leur département, qui lui-même peut adhérer à la section apicole de l'Organisme à vocation sanitaire (SA-OVS) régional (le GDS Aquitaine), partenaire de l'État dans la surveillance, la prévention et la lutte contre les pathologies. Les apiculteurs peuvent aussi adhérer à la SA-OVS, soit directement, soit via l'Adaaq (Association de développement de l'apiculture en Aquitaine) qui adhère à cette section apicole. La fusion des trois régions Aquitaine, Limousin et Poitou-Charentes va probablement renforcer cette complexité. Aussi le mode de prise de décision au sein de la SA-OVS peut-il être relativement compliqué, conduisant par exemple à des décisions en faveur de telle ou telle catégorie d'apiculteurs. Les dynamiques particulières d'évolution des pathogènes peuvent d'ailleurs rendre l'anticipation des effets de telle ou telle décision relativement opaque.

Notre ambition se limite à montrer que le choix du processus de représentation démocratique au niveau de la région a un impact direct sur l'évolution du nombre de colonies viables. Les différents modes de représentation lors d'un vote pourraient être, par exemple : une voix par apiculteur (quel que soit le nombre de colonies détenues) ; une voix par colonie (apiculteur ayant 1000 colonies $=1000$ voix) ; seuls décident les apiculteurs de plus de $n$ colonies, avec pour eux une voix par apiculteur ; une voix par colonie, mais au nombre plafonné à $n$ voix pour un apiculteur ; et deux cas limites : l'État décide seul dans son propre intérêt ; aucune décision, personne n’impose rien.

Dans notre modèle chaque apiculteur prend en compte les produits qu'il vend, ainsi qu'éventuellement les aménités liées à l'apiculture. L'État a des missions régaliennes de lutte contre les pathologies; mais à son niveau, nous intégrons uniquement dans notre modèle le nombre de colonies sur lesquelles il perçoit une taxe, la vente de miel (pour son effet indirect sur les activités économiques, les taxes et impôts) ainsi que les aménités liées à la présence d'abeilles (effet pollinisateur). La variable de commande pour l'État est le mode de prise de décision au sein de la SA-OVS de la région. On se trouve ainsi dans le cadre d'un 
modèle principal-agent (voir par exemple Laffont et Martimort [2002]), avec divergence d'intérêt entre les apiculteurs (les agents, pris individuellement) et l'État (le principal). Étant donné que l'État perçoit des taxes et impôts sur les activités des apiculteurs et qu'il est sensible aux différentes externalités liées à cette activité, il y a aussi une divergence d'intérêt, mais dans une moindre mesure, entre l'État et la SA-OVS (qui cherche seulement à lutter contre les pathologies), ou plus généralement entre l'État et l'ensemble des apiculteurs qui forment alors un seul agent. On pourrait ainsi introduire différents niveaux de relations principal-agent entre ces entités, mais on laissera cette extension pour des travaux futurs.

En examinant simplement la répartition des apiculteurs en fonction de la taille de leur rucher (voir figure $n^{\circ} 1$ et tableau $\mathrm{n}^{\circ} 1$ ), et si on suppose une segmentation entre petits, moyens et grands apiculteurs qui constituent alors trois collèges pour la prise de décision, éventuellement associés à l'État, on comprend que lorsque les intérêts des uns et des autres divergent :

- si le mode de décision est une voix par apiculteur, les petits apiculteurs emportent la décision, quelle que soit la position des moyens et des grands (modalité de décision A) ;

- si le mode de décision est une voix par colonie, les grands apiculteurs emportent la décision, quelle que soit la position des petits et des moyens (modalité B) ;

- si seuls décident les apiculteurs de plus de $n$ colonies, et qu'alors pour eux un apiculteur = une voix, pour que les grands apiculteurs emportent seuls la décision, comme ils sont au nombre de 63, il peut leur être adjoint au maximum 62 apiculteurs moyens qui ne changeront pas la décision. L'apiculteur à la limite est le $125^{\text {ième }}$ apiculteur de la région, dans l'ordre décroissant de la taille des ruchers. La base de données révèle qu'il possède 100 ruches. La limite $n$ peut ainsi être fixée à $n=101$. Pour $n$ supérieur ou égal à cette valeur, les grands apiculteurs emportent seuls la décision (modalité C). Pour $n$ inférieur à cette valeur, les petits et moyens emportent la décision (modalité D). D'autres cas peuvent être envisagés : par exemple pour que les moyens, seuls, puissent emporter la décision, on peut leur adjoindre un 
nombre $N$ de petits tel que $N+$ nombre de grands < nombre de moyens, c'est-à-dire $N<245-63$, soit $N<182$. La taille de la ruche limite est celle de l'apiculteur de rang (par ordre croissant de taille) égal à 1199 (nombre de petits apiculteurs) - 182 $=1017$; soit $n=10$ ruches. Autrement dit, pour $11 \leq n \leq 25$ (cette seconde limite permettant de n'évincer aucun apiculteur moyen), les apiculteurs moyens décident seuls (modalité E).

D'autres modalités de décision (une voix par colonie mais avec une limite de $n$ voix ; seuls décident les apiculteurs de plus de $n$ colonies...) pourraient aussi être envisagées, et comparées aux précédentes. Bien entendu la situation n'est pas toujours aussi tranchée, et il n'y a pas nécessairement d'opposition des apiculteurs segmentés sur la base de la taille de leur rucher. Néanmoins, on ne peut occulter qu'il s'agit d'une dimension importante dans la prise de décision collective, au-delà d'une opposition entre apiculteurs professionnels et de loisir.

Cette divergence d'intérêt entre les apiculteurs, concernant la mise en ouvre de traitements contre les pathologies, peut en particulier provenir à la fois des coûts de ces traitements, et des bénéfices qui en sont attendus. Concernant les coûts, on peut schématiquement les séparer en coûts fixes et coûts variables. Les coûts fixes comprennent en particulier les coûts d'acquisition d'information, par exemple apprendre à reconnaître une colonie atteinte de la pathologie, ou apprendre que certains fournisseurs de colonies ou de miel de nourrissage peuvent être contaminés. Ramenés au nombre de colonies, ils pourront être particulièrement élevés pour les petits ruchers, alors qu'ils resteront faibles pour les plus grands. D'autres sources de divergence d'intérêt sont les modalités de calcul des taxes et impôts divers, et plus généralement les bénéfices et utilités retirés de l'apiculture.

\section{Modèle de diffusion du pathogène : l'aléa}

La loque américaine est une pathologie virulente, réputée légalement contagieuse et classée parmi les dangers sanitaires de catégorie 1 , due à une bactérie (Paenibacillus larvae) qui provoque la mort des larves d'abeilles, et se diffuse par des spores 
particulièrement résistants. La contamination se fait de rucher à rucher, avec une transmission de spores par les abeilles (les colonies affaiblies se font voler le miel par des abeilles étrangères qui rapportent des spores dans leur colonie d'origine), ou par exemple par les apiculteurs, d'une colonie à l'autre de leur rucher. La contamination peut être aussi due à des contacts entre abeilles par exemple lors du butinage, ou au nourrissage des abeilles l'hiver, avec des miels contenant des spores, ou encore à l'achat, pour compléter un rucher, de colonies qui se révéleront être infestées. Les spores permettent à cette maladie de perdurer, malgré sa virulence et la mortalité induite sur les populations hôtes. De manière à pouvoir tenir compte de cette dynamique, le modèle travaille sur un horizon relativement long.

La détection n'est pas facile, et souvent les apiculteurs amateurs peu formés doivent se faire aider s'ils ont un doute. Les colonies malades mais encore fortes peuvent être transvasées dans une ruche indemne. L'ancienne ruche doit être désinfectées ou détruite. Les colonies jugées trop faibles sont asphyxiées puis incinérées. La loque américaine peut être combattue de différentes manières, aucune n'étant totalement efficace, mais chacune contribuant à freiner sa diffusion.

Il n'a pas été fait de déclaration de loque américaine en 2014 en Aquitaine. Mais en 2012-2013, le programme européen Epilobee (voir Chauzat et al. [2014]) a permis d'estimer un ordre de grandeur de l'étendue possible de la maladie : le taux de ruchers dont on a eu confirmation qu'ils étaient cliniquement atteints a été entre $2 \%$ et $12 \%$ sur les six départements français objets de l'étude (dont aucun en Aquitaine). Le rapport Gerster ([2012], proposition P3) insistait déjà sur l'intérêt de mettre en place un système d'épidémio-surveillance sur les loques, ainsi que des prophylaxies sanitaires contre la loque américaine.

Différents modèles permettent de représenter la diffusion d'un pathogène aussi complexe que celui-ci. Tout d'abord on peut distinguer les modèles stratifiés (par exemple Ball et Neal [2002] ; ou pour une application à l'apiculture, Fries et Camazine [2001]), avec diffusion du pathogène à l'intérieur de sous-ensembles (ici par exemple à l'intérieur des colonies) diffé- 
rente de la diffusion d'un sous-ensemble à l'autre. Il serait possible de considérer que l'on a une population stratifiée à trois niveaux : à l'intérieur d'une colonie, entre colonies d'un même rucher et enfin d'un rucher à l'autre. Mais pour atteindre notre objectif nous sommes seulement amenés à distinguer les colonies qui appartiennent à de grands ruchers de celles qui appartiennent à des ruchers moyens ou petits, et affectons des facteurs de diffusion possiblement différents pour chacun de ces types.

Les modèles de diffusion de pathologie non explicitement spatialisés peuvent, à leur tour, être divisés en différentes catégories : par exemple les modèles SIR ( $S$ pour susceptibles : la population non atteinte ; I pour infectée ; R pour recovery : la population ayant subi la pathologie et non susceptible de l'attraper à nouveau ; voir par exemple Murray [2002] chap. 10), ou les modèles SIS, lorsque cette dernière catégorie $\mathrm{R}$ n'existe pas et que les individus après avoir été infectés sont susceptibles d'être à nouveau atteints (voir par exemple Hethcote [1989] et Allen et Burgin [2000]). Dans notre travail concernant la loque américaine nous sommes amenés à mettre en place un modèle intermédiaire entre SIR et SIS, dans lequel existent deux populations : les colonies S (susceptibles), les colonies I (infectées) lesquelles peuvent rejoindre les colonies $S$ ou laisser place à de nouvelles colonies $S$, mais une fois seulement qu'elles auront été détectées et transvasées ou remplacées, ce qui demande un délai de quelques années. Cette notion de délai (ou retard), peut conduire à des phénomènes d'oscillation des populations, ou, selon les paramètres, à une convergence vers un état stationnaire (voir par exemple Rapaport et al. [2003]), éventuellement après quelques oscillations amorties. Nous l'introduisons toutefois ici car la détection de la présence de la loque américaine dans les colonies n'est pas toujours faite au plus vite par les détenteurs de petits ruchers, faute d'information sur les symptômes de la pathologie. En outre, les temps de traitement des ruches ou des colonies ne peuvent être négligés ici, et une production normale de miel n'est possible que quelques années après la détection et le traitement. Les équations du modèle de diffusion du pathogène sont présentées en annexe $\mathrm{n}^{\circ} 1$. 
Nous avons vérifié que le modèle ne présentait pas d'instabilité par rapport aux différents paramètres (par exemple une variation de $10 \%$ des éléments de la matrice $K$ entraîne une variation inférieure à $10 \%$ des résultats sur les utilités de chaque catégorie d'apiculteur et de l'État; il en est de même pour le prix du miel, la valeur d'existence d'une colonie, les différentes taxes et cotisations, le coût du traitement, les coefficients d'actualisation, le taux d'infestation au départ). Le paramètre le plus sensible est le temps de détection de la maladie, et c'est celui sur lequel porteront les moyens de lutte étudiés dans les sections suivantes.

\section{Premières simulations}

\section{Sans le pathogène}

Cette situation permet de mesurer l'utilité inter-temporelle d'un scénario de référence hypothétique. Ici on suppose que la loque américaine n'a pas atteint la région. Aucune colonie n'est infestée et toutes restent dans l'état "susceptible». Les courbes d'évolution des effectifs susceptibles restent plates, à leur maximum. Tous les paramètres sont fixés à leur valeur donnée en annexe 2, sauf $J_{1,1}$ qui est un vecteur nul (pas d'infestation au départ).

La valeur des utilités actualisées est alors donnée dans le tableau $n^{\circ} 2$ (exprimée en millions d'euros; par exemple pour la première colonne : utilité de l'ensemble des grands ruchers ; en valeur annualisée ${ }^{3}$.

Tableau 2

\section{Utilités dans la situation sans pathogène}

\begin{tabular}{|c|c|c|c|c|}
\hline grands & moyens & petits & ensemble apiculteurs & État \\
\hline 2,95 & 1,45 & 0,57 & 4,97 & 2,98 \\
\hline
\end{tabular}




\section{Avec le pathogène, sans mesure complémentaire de lutte}

On suppose qu'au départ $(t=1)$ toutes les colonies étaient dans la catégorie susceptible, comme précédemment, excepté une seule colonie parmi les petits ruchers qui est infestée (soit $0,0834 \%$ des colonies des petits ruchers). Le tableau $n^{\circ} 3$ présente les résultats obtenus en valeurs annualisées.

\section{Tableau 3}

\section{Utilités dans la situation avec pathogène, mais sans mesure de lutte}

\begin{tabular}{|c|c|c|c|c|}
\hline grands & moyens & petits & ensemble apiculteurs & État \\
\hline 2,09 & 1,11 & 0,356 & 3,56 & 2,16 \\
\hline
\end{tabular}

On constate ainsi une perte conséquente des utilités pour toutes les catégories de ruchers. La figure $n^{\circ} 2$ illustre l'évolution du cheptel sain et des utilités.

Dans cette figure, toutes les échelles des ordonnées partent de zéro, de manière à éviter des effets purement visuels qui conduiraient à mésestimer certaines variations. Elle représente dans la colonne de gauche, de haut en bas, l'évolution des effectifs susceptibles pour un grand rucher représentatif (premier graphique), c'est-à-dire dont l'effectif est la moyenne de sa catégorie ; puis pour un rucher moyen représentatif (deuxième graphique) ; et enfin pour un petit rucher représentatif. Au départ la pathologie est peu présente, même parmi les petits ruchers, puis elle se diffuse rapidement pour atteindre ensuite les deux autres catégories. Pour les petits ruchers une amorce d'oscillation est visible, due à l'effet retard présenté précédemment. On remarque aussi que les effectifs de "susceptibles " finissent par converger vers une valeur stationnaire assez basse pour les trois types de rucher. 


\section{Figure 2}

\section{Situation de référence}
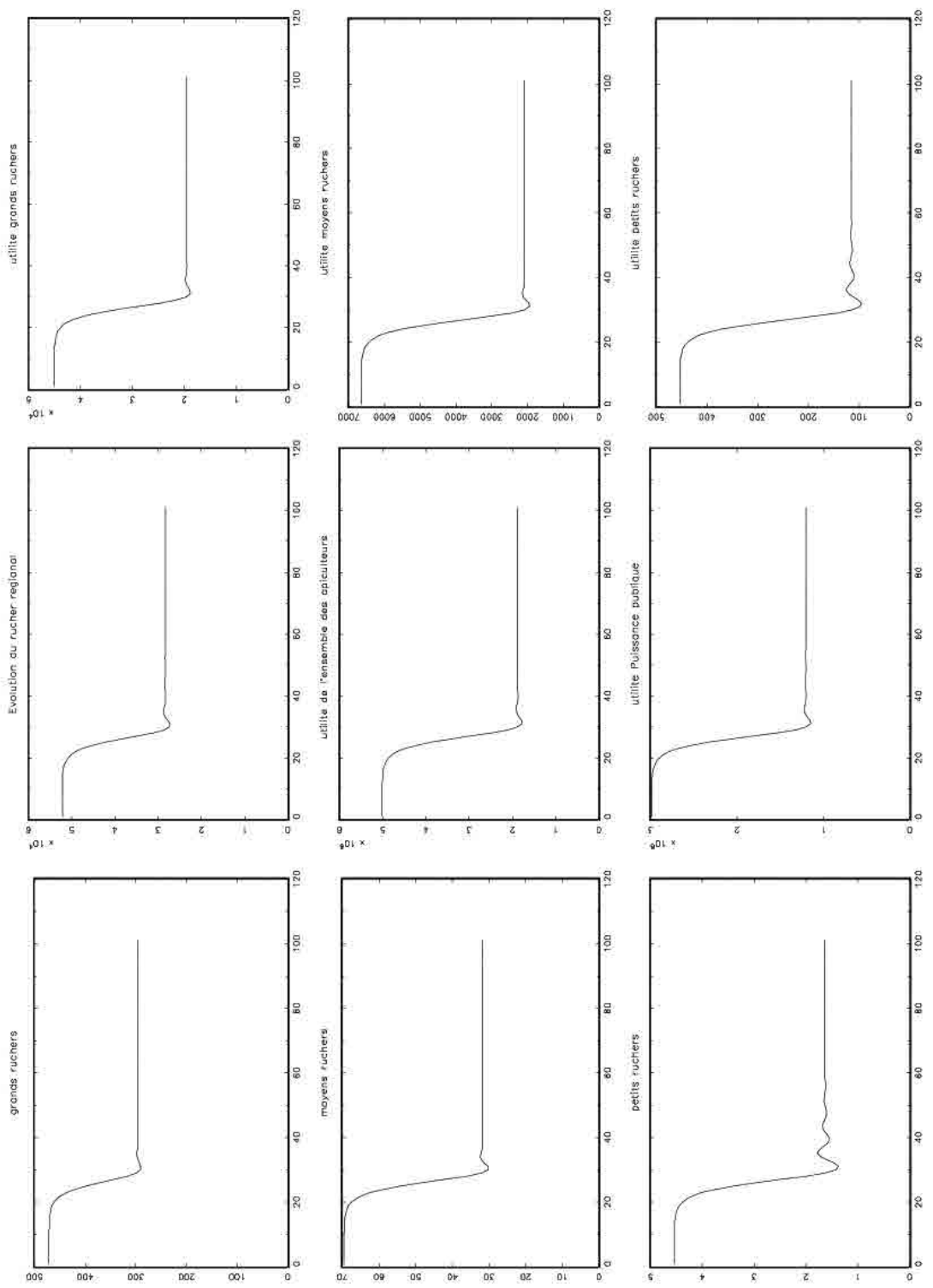

Note : voir le texte en section " avec pathogène, sans mesure complémentaire de lutte ". 
Dans la colonne de droite, de haut en bas, est représentée l'utilité pour un grand rucher représentatif, un moyen rucher, puis un petit rucher. L'importance de cette perte ne se retrouve pas dans la valeur actualisée du tableau précédent (tableau $\mathrm{n}^{\circ} 3$ ), qui, lui, tient compte de l'ensemble de la chronique, et en conséquence de l'absence de perte les premières années.

Dans la colonne du milieu, sur le graphique du haut est représentée l'évolution du rucher régional, à savoir l'ensemble de toutes les colonies de la région, à partir de l'évolution du rucher moyen de chaque catégorie et du nombre de ruchers dans chaque catégorie. La perte totale de colonies saines est au final de l'ordre de $50 \%$. Le second graphique, au centre, représente l'utilité de l'ensemble des apiculteurs, à savoir la somme des utilités de chaque rucher représentatif de sa catégorie, multipliée par le nombre de ruchers dans chaque catégorie. Le troisième graphique représente l'évolution de l'utilité de l'État, telle que définie précédemment.

Les divergences d'intérêt entre les apiculteurs peuvent provenir de la perte relative de leur utilité (plus forte par exemple chez les apiculteurs moyens que chez les grands), mais aussi des moyens de lutte contre la pathologie qui peuvent être mis en œuvre et de la répartition des coûts associés.

\section{Moyens de lutte : modification de la détection}

Partons de la situation de référence présentée précédemment (section avec le pathogène, sans mesure complémentaire de lutte), et supposons qu'il soit possible, sans coût, d'accélérer la détection de la loque américaine chez les seuls apiculteurs moyens (cette détection restant identique chez les grands (rapide) et chez les petits apiculteurs (lente) ; à savoir $\theta_{i}=(1,2,5)^{\prime}$ au lieu de $\left.(1,3,5)^{\prime}\right)$ à partir de la date ${ }^{4} 20$, et calculons l'évolution des utilités sur un horizon de 5 ans, horizon retenu pour la prise de décision, en fonction de la modification de la rapidité de détection, dans un premier temps sans tenir compte des coûts associés 
à cette amélioration. Les résultats sont présentés dans le tableau $\mathrm{n}^{\circ} 4$, en valeur cumulée actualisée avec un taux de $4 \%$ (cumul entre les années 20 et 24), puis transformés en valeur annuelle.

\section{Tableau 4}

Amélioration à la date 20 de la rapidité de détection de la loque américaine et augmentation induite des utilités sur cinq ans, en valeur annualisée sans tenir compte des coûts induits par cette amélioration

\begin{tabular}{|c|c|c|c|c|c|c|}
\hline $\begin{array}{c}\text { Utilités } \\
\text { en millions d'€ }\end{array}$ & $\boldsymbol{\theta}_{i}$ & grands & moyens & petits & $\begin{array}{c}\text { Ensemble } \\
\text { apiculteurs }\end{array}$ & État \\
\hline $\begin{array}{c}\text { Détection } \\
\text { de référence }\end{array}$ & $(1,3,5)$ & 2,6349 & 1,4878 & 0,4937 & 4,6164 & 2,7537 \\
\hline $\begin{array}{c}\text { Amélioration } \\
\text { moyens }\end{array}$ & $(1,1,5)$ & 2,6621 & 1,5174 & 0,4987 & 4,6781 & 2,7912 \\
\hline $\begin{array}{c}\text { Amélioration } \\
\text { petits }\end{array}$ & $(1,3,1)$ & 2,6374 & 1,4892 & 0,4989 & 4,6256 & 2,7594 \\
\hline $\begin{array}{c}\text { Amélioration } \\
\text { moyens et petits }\end{array}$ & $(1,1,1)$ & 2,6646 & 1,5188 & 0,5040 & 4,6874 & 2,7969 \\
\hline
\end{tabular}

Prenons la première mesure qui consiste en une amélioration du temps de détection chez les moyens. Et supposons maintenant que le coût annuel d'une telle mesure est de $C$. Dans ce qui suit, on suppose que la décision est prise par des représentants des trois collèges de décideurs, les grands, les moyens et les petits apiculteurs, et nous examinons l'intérêt collectif de chacun de ces collèges. Dans la suite du document nous ferons une analyse plus fine à partir de l'intérêt individuel des membres de ces différents collèges. Dans ce cadre, on peut imaginer que la répartition de ce coût est faite par exemple selon deux modalités : soit au prorata du nombre de colonies, soit à égalité entre les apiculteurs. On notera que généralement les mesures de lutte contre les pathologies incluent un coût fixe par rucher (par exemple acquisition d'un savoir-faire pour détecter une pathologie) ou un coût variable en fonction du nombre de colonies (par exemple visite systématique des colonies). Les deux cas que nous présentons sont ainsi deux cas limites. 
A/ Répartition du coût au prorata des colonies (COL)

Sachant que les grands ruchers représentent $56,90 \%$ des colonies $(29,699 / 52,199)$, les moyens $32,68 \%$ et les petits $10,42 \%$, une analyse coût-bénéfice (ACB) chez les grands apiculteurs donnera un résultat positif si, et seulement si, leur gain en utilité $(2662,1-2634,9=27,2 \mathrm{k} €)$ est tel que $27,2 \mathrm{k} €-0,5690 \mathrm{C}>0$; Soit $\mathrm{C}<47,8 \mathrm{k} €$. Pour les moyens, un même calcul conduit à ce que l'ACB est positive si, et seulement si, $\mathrm{C}<90,9 \mathrm{k} €$ et pour les petits $\mathrm{C}<48,1 \mathrm{k} €$.

Autrement dit, si le coût annuel $C<47,8 \mathrm{k} €$, tous sont bénéficiaires et il y a unanimité pour mettre en ouvre la mesure, si ce sont les apiculteurs qui décident. Ils sont en cela appuyés par l'État dont l'ACB de la mesure est, elle aussi, positive (son bénéfice est sur le même laps de temps de 2,7912-2,7537 $10^{6} €$ $=37,5 \mathrm{k} €$ ). Cet intervalle est ainsi extensible jusqu'à $C<85,3 \mathrm{k} €$ $(=47,8+37,5)$.

Si $47,8<C<48,1 \mathrm{k} €$, seuls les apiculteurs moyens et petits bénéficient de la mesure. Celle-ci sera adoptée si eux seuls décident : ce sera le cas si la modalité de décision est $\mathrm{A}, \mathrm{D}$ ou $\mathrm{E}$. La participation de l'État peut étendre cet intervalle jusqu'à la valeur maximale de $85,6 \mathrm{k} €$.

Si $48,1<C<90,9 \mathrm{k} €$, seuls les apiculteurs moyens sont bénéficiaires de la mesure qui sera adoptée si eux seuls décident. Ce sera le cas si la modalité de décision est E. La participation de l'État peut étendre cet intervalle jusqu'à la valeur maximale de $128,4 \mathrm{k} €$.

Si 90,9 $<C<128,4 \mathrm{k} €$, aucun apiculteur n'est bénéficiaire, mais s'il y a prise en charge partielle du coût par l'État, le même raisonnement que précédemment s'applique, et la mesure peut quand même être adoptée.

Enfin pour $C>128,4 \mathrm{k} €$, la mesure est trop onéreuse pour être amortie sur cet horizon de 5 ans.

B/ Répartition du coût au prorata des ruchers (RUC) : le détail des calculs est reporté en annexe nº3. 
$\mathrm{C} / \mathrm{Si}$ l'on cumule les deux ensembles de possibilités de règles de décision pour mettre en œuvre la mesure, présentés sections $\mathrm{A}$ et $\mathrm{B}$ précédentes, on obtient le tableau $\mathrm{n}^{\circ} 5$ (avec les gagnants ou perdants en termes d'utilité : $G$ pour grands, $M$ pour moyens, $P$ pour petits, E pour État ; et selon les modalités de répartition du coût : COL pour une répartition au prorata des colonies, RUC au prorata des ruchers).

Au final, et sans même faire une évaluation similaire pour les autres hypothèses concernant l'amélioration possible de la détection de la pathologie présentées dans le tableau $\mathrm{n}^{\circ} 4$, il ressort de ces calculs que les modalités de décision au sein de la SA-OVS sont un facteur essentiel pour la dynamique d'évolution du cheptel sain et des utilités des apiculteurs et de l'État. Il est clair que ces résultats de simulations ne peuvent être utilisés en dehors de cet objectif ; nous n'avons pas tenu compte, par exemple, de la variabilité de la propagation du pathogène en fonction de la géographie ni de la densité des ruchers dans certaines zones. En conséquence, il ne s'agit pas de valeurs prédictives, mais de l'illustration d'un résultat qualitatif.

Tableau 5

Modalités de décision conduisant à l'acceptation de la mesure proposée à la troisième ligne du tableau $n^{\circ} 4$ (amélioration de la détection chez les moyens)

\begin{tabular}{|l|l|l|l|l|l|}
\hline Coût C en $10^{3} €$ & $\begin{array}{l}\text { Extensible } \\
\text { à }\left(^{*}\right)\end{array}$ & $\begin{array}{l}\text { Répartition } \\
\text { du coût }\end{array}$ & $\begin{array}{l}\text { Ensembles } \\
\text { gagnants }\end{array}$ & $\begin{array}{l}\text { Ensembles } \\
\text { perdants }\end{array}$ & $\begin{array}{l}\text { Modalités } \\
\text { de décision, } \\
\text { pour que la } \\
\text { mesure soit } \\
\text { acceptée }\end{array}$ \\
\hline $\mathrm{C}<6,30$ & $\mathrm{C}<43,8$ & $\begin{array}{l}\text { RUC ou } \\
\mathrm{COL}\end{array}$ & $\mathrm{G}, \mathrm{M}, \mathrm{P}, \mathrm{E}$ & - & toutes \\
\hline \multirow{2}{*}{$6,30<\mathrm{C}<47,8$} & $\mathrm{C}<85,3$ & $\mathrm{RUC}$ & $\mathrm{G}, \mathrm{M}, \mathrm{E}$ & $\mathrm{P}$ & $\mathrm{B}, \mathrm{C}, \mathrm{E}$ \\
\cline { 3 - 6 } & $\mathrm{COL}$ & $\mathrm{G}, \mathrm{M}, \mathrm{P}, \mathrm{E}$ & & toutes \\
\hline \multirow{2}{*}{$47,8<\mathrm{C}<48,1$} & $\mathrm{C}<85,6$ & $\mathrm{RUC}$ & $\mathrm{G}, \mathrm{M}, \mathrm{E}$ & $\mathrm{P}$ & $\mathrm{B}, \mathrm{C}, \mathrm{E}$ \\
\cline { 3 - 6 } & $\mathrm{COL}$ & $\mathrm{M}, \mathrm{P}, \mathrm{E}$ & $\mathrm{G}$ & $\mathrm{A}, \mathrm{D}, \mathrm{E}$ \\
\hline \multirow{2}{*}{$48,1<\mathrm{C}<90,9$} & $\mathrm{C}<128,4$ & $\mathrm{RUC}$ & $\mathrm{G}, \mathrm{M}, \mathrm{E}$ & $\mathrm{P}$ & $\mathrm{B}, \mathrm{C}, \mathrm{E}$ \\
\cline { 3 - 6 } & $\mathrm{COL}$ & $\mathrm{M}, \mathrm{E}$ & $\mathrm{G}, \mathrm{P}$ & $\mathrm{E}$ \\
\hline
\end{tabular}


186 Jean-Philippe Terreaux

\begin{tabular}{|l|l|l|l|l|l|}
\hline \multirow{2}{*}{ Coût C en $10^{3} €$} & $\begin{array}{l}\text { Extensible } \\
\text { à (*) }\end{array}$ & $\begin{array}{l}\text { Répartition } \\
\text { du coût }\end{array}$ & $\begin{array}{l}\text { Ensembles } \\
\text { gagnants }\end{array}$ & $\begin{array}{l}\text { Ensembles } \\
\text { perdants }\end{array}$ & $\begin{array}{l}\text { Modalités } \\
\text { de décision, } \\
\text { pour que la } \\
\text { mesure soit } \\
\text { acceptée }\end{array}$ \\
\hline \multirow{2}{*}{$90,9<\mathrm{C}<558,9$} & $\mathrm{C}<596,4$ & $\mathrm{RUC}$ & $\mathrm{G}, \mathrm{M}, \mathrm{E}$ & $\mathrm{P}$ & $\mathrm{B}, \mathrm{C}, \mathrm{E}$ \\
\cline { 3 - 6 } & $\mathrm{COL}$ & - & $\mathrm{G}, \mathrm{M}, \mathrm{P}, \mathrm{E}$ & aucune \\
\hline $558,9<\mathrm{C}<$ \\
650,7 & $\mathrm{C}<688,2$ & $\mathrm{RUC}$ & $\mathrm{G}, \mathrm{E}$ & $\mathrm{M}, \mathrm{P}$ & $\mathrm{B}, \mathrm{C}$ \\
\hline & $\mathrm{COL}$ & - & $\mathrm{G}, \mathrm{M}, \mathrm{P}, \mathrm{E}$ & aucune \\
\hline $650,7<\mathrm{C}$ & & $\begin{array}{l}\mathrm{RUC} \text { ou } \\
\mathrm{COL}\end{array}$ & - & $\mathrm{G}, \mathrm{M}, \mathrm{P}, \mathrm{E}$ & aucune \\
\hline
\end{tabular}

Note $\left(^{*}\right)$ : selon la participation de l'État, d'un montant maximal de 37,5 k€.

D/ Décisions individuelles des apiculteurs : lorsque le collège des petits apiculteurs est globalement gagnant, et en conséquence favorable à la mesure étudiée, cela ne signifie pas que chacun des membres de ce collège est lui-même gagnant. Sachant que les petits apiculteurs ont entre 1 et 25 colonies, il est clair que si une mesure peut être acceptable pour les plus grands d'entre eux, suite à une analyse coût-bénéfice, elle ne l'est pas nécessairement pour les plus petits, ou inversement. Il est possible alors de refaire, au niveau des seuls petits apiculteurs, le même travail que le précédent fait au niveau de l'ensemble des apiculteurs. On arriverait ainsi à déterminer en fonction du coût des mesures et de la manière de répartir ce coût entre les apiculteurs, les modalités de représentation de chacun des petits apiculteurs qui conduiraient à ce que le collège de petits apiculteurs soit globalement favorable ou défavorable à l'adoption de la mesure.

\section{Extensions et conclusion}

Nous visons avant tout la simplicité de la représentation et n'avons pas pour but de prédire quantitativement les évolutions futures du nombre de colonies, mais simplement d'indiquer, selon les modalités de décision mises en œuvre, l'orientation 
possible de ces évolutions. Aussi ce modèle reste-t-il avant tout qualitatif et non pas quantitatif, même s'il est fondé sur des modèles éprouvés pour ce type de diffusion et s'il est paramétré au mieux des connaissances actuelles. Nous avons vu que le mode de décision au sein de l'OVS est essentiel à la mise en place ou non de mesures de lutte et, partant, à l'évolution de la pathologie. La loque américaine est une pathologie importante par sa virulence, sa capacité à se transmettre horizontalement (d'une colonie à l'autre) et verticalement (par essaimage) et par les dégâts occasionnés. En outre, ce travail pourrait être étendu à d'autres pathologies impactant l'apiculture (voir par exemple FAO [2006] ou encore Genersch et al. [2010]), et notamment la loque européenne (due à deux bactéries différentes), la varroase (Varroa destructor), la nosémose (Nosema apis et Nosema ceranae) etc. mais au prix de modifier le modèle de diffusion de la pathologie. Il pourrait aussi être étendu pour aborder la lutte contre tout autre nuisible, tels le frelon asiatique (Vespa velutina), à partir du moment où les intérêts des apiculteurs divergent quant aux moyens à employer et qu'il existe des externalités aux actions de chacun.

Concernant la loque américaine, nous aurions pu considérer d'autres types de répartition des coûts que l'égalité entre les ruchers ou au prorata du nombre de colonies, ou prendre en compte des mesures incluant ces deux répartitions. Nous aurions pu aussi travailler sur les mesures correspondant aux autres lignes du tableau $\mathrm{n}^{\circ} 4$ ou sur d'autres types de mesures, conduisant à la variation d'autres paramètres de l'évolution de la pathologie. Il aurait été possible aussi de partir non pas de valeurs déterministes des paramètres, mais d'une distribution de probabilité de ces paramètres entre les différents apiculteurs. L'aversion au risque des apiculteurs aurait pu être introduite. Une représentation de l'approche spatiale de la diffusion des pathogènes était aussi envisageable.

Nous avons supposé ici que tous les paramètres et toutes les motivations étaient connus de tous. Or, pour ces différentes maladies, l'OVS peut avoir à prendre des décisions dans le cadre d'informations qui sont incomplètes, parfois biaisées, ou véri- 
fiables seulement partiellement (voir Green et Laffont [1986]). Ces informations peuvent concerner pour chaque apiculteur ses coûts, les variations d'utilité induites par la maladie ou encore le niveau d'effort opéré. L'importance du problème varie selon les pathologies, par exemple l'apiculteur pouvant supporter des coûts de traitement plus importants que ce qu'il en retire en termes d'utilité, n'étant de ce fait guère enclin à révéler une information sur la présence du pathogène qui le pénaliserait. Nous avons vu que dans ce cadre pouvaient être envisagés d'autres processus de décision qu'un système de vote (par exemple des décisions autoritaires de l'État, avec de possibles difficultés liées à une acceptabilité moindre). Une décision par vote semble plus empreinte de démocratie, qui reste le mode de gouvernement conçu depuis l'Antiquité comme "le règne de l'égalité et de la liberté " (Zarka [2010]). Mais nous avons montré ici comment les défauts d'un tel système, que Platon dénonçait déjà, pouvaient conduire à des situations peu souhaitables.

En réalité, il y a une multiplicité de décisions à prendre au fil du temps par l'organisme en charge du problème, et l'on ne peut vouloir tout représenter à travers des modèles, si l'on veut que ces derniers puissent donner des résultats interprétables. Et il ne s'agit pas de mettre en place un mode de décision qui soit fonction du problème traité, de manière à arriver à chaque fois à la décision souhaitée a priori par le responsable. Mais les résultats obtenus, qui restent qualitatifs, montrent par exemple que les apiculteurs ayant de grands ruchers seront quasiment toujours favorables ( $c f$. les valeurs très élevées correspondantes dans le tableau $\mathrm{n}^{\circ}$ 5) à des mesures dont le coût sera réparti, à parts égales, entre les différents ruchers, puisqu'ils retirent généralement une grande utilité de l'impact de ces mesures, alors que l'analyse coût-efficacité n'est pas aussi favorable chez les apiculteurs de loisir. Ce sera le cas des mesures impliquant des coûts fixes par rucher, comme les coûts d'information, de surveillance, d'équipement spécifique, de réseau d'approvisionnement en matériel ou en essaims certifiés etc. À l'inverse, on aurait pu penser que les apiculteurs de loisir bénéficiaient des mesures dont les coûts étaient essentiellement proportionnels au nombre 
de colonies, coûts alors collectivement principalement supportés par les apiculteurs professionnels. Nous ne l'avons pas constaté sur les deux exemples de mesures envisagées, l'explication pouvant provenir de la complexité induite d'une part par l'épidémiologie de la loque américaine, d'autre part par le contexte économique et fiscal que nous avons représenté ici.

La question de l'organisation de la démocratie, et la représentation de différents collèges d'apiculteurs (de loisir, professionnels, pluri-actifs) dans la structure de lutte sanitaire sont ainsi un point essentiel, aux côtés de la connaissance épidémiologique des pathologies et de la connaissance des différents aspects techniques. Cette organisation ne peut être sousestimée, ni considérée comme indifférente et sans conséquence sur les résultats ; elle permet de prendre en considération, et sans s'en étonner, les arguments des opposants et des partisans de différentes actions de lutte, et éventuellement de mettre en œuvre, pour les perdants, de justes mesures compensatoires.

Enfin cette question de la représentation démocratique peut être généralisée aux autres structures à vocation sanitaire, notamment dans la lutte contre différentes épizooties dans le monde agricole.

L'auteur tient à remercier particulièrement les relecteurs, notamment de la RFE, pour leurs remarques pertinentes qui ont conduit à développer les différentes parties de cet article, et à en améliorer la présentation. Il reste bien sûr seul responsables des imprécisions et erreurs qui auraient pu subsister.

Jean-Philippe Terreaux est ingénieur en chef des Ponts, des eaux et des forêts à Irstea, l'Institut national de recherche en sciences et technologies pour l'environnement et l'agriculture. Adresse : Irstea, UR ETBX, 50 avenue de Verdun, Gazinet, 33612 Cestas cedex, tél : 0557890164.

Email: jean-philippe.terreaux@irstea.fr 


\section{Annexes}

\section{Annexe 1 \\ Modèle de diffusion de la pathologie}

$t$ représente le temps; $H$ est l'horizon des calculs.

$i$ est un indice indiquant la catégorie d'appartenance du rucher considéré : $i=1$ : grand rucher ; $i=2$ : moyen ; $i=3$ : petit.

$S_{i}(t)$ représente le nombre de colonies de catégorie $i$, à la date $t$, qui sont "susceptibles", c'est-à-dire qui n'ont pas la pathologie. $\theta_{i}$ est le temps nécessaire à la détection de la pathologie, et au rétablissement de la colonie atteinte ou à son remplacement par une colonie saine.

$J_{i, t}$ est le vecteur de taille $\theta_{i} \mathrm{du}$ nombre de colonies infestées à la date $t$, pour la catégorie de rucher $i$. La coordonnée $k=1,2, \ldots$ $\theta_{i}$ de $J_{i, t}$, notée $J_{i, t, k}$,représente le nombre de colonies infestées depuis $1,2, \ldots \theta_{\text {i années. }}$

$K$ est la matrice de $\mathfrak{l}^{3} \mathrm{x} \mathfrak{l}^{3}$ de propagation de la pathologie entre les petits, moyens et grands ruchers.

Le nombre de nouveaux infestés à la date $t+1$, pour chaque catégorie, est supposé proportionnel au nombre de susceptibles dans la catégorie considérée, et aussi au nombre total des infestés pour l'ensemble des catégories, à la date $t$.

Le nombre total d'infestés dans la population des colonies de la catégorie $i$ à la date $t$ est :

$$
\sum_{j=1}^{\theta_{i}} J_{i, t, j}
$$

Et le nombre de nouveaux infestés de la catégorie $i$, à la date $t+1$, s'écrit :

$$
J_{i, t, j}=\sum_{n=1}^{3}\left(K(i, n) \cdot S_{i}(t) \cdot\left(\sum_{j=1}^{\theta_{i}} J_{n, t, j}\right)\right)
$$


Il ne peut y avoir cependant plus de colonies nouvellement infestées que de colonies initialement susceptibles, et de ce fait $J_{i, t+1,1}$ doit rester inférieur à $S_{i}(t)$. Au total :

$$
J_{i, t+1,1}=\operatorname{Max}\left\{\sum_{n=1}^{3} k(i, n) \cdot S_{i}(t) \cdot\left(\sum_{j=1}^{\theta_{i}} J_{n, t, j}\right), \quad S_{i}(t)\right\}
$$

Ce nombre constitue la première coordonnée du vecteur $J_{i, t+1}$ et, pour $k>1, J_{i, t+1, k}=J_{i-1, t, k-1}$, autrement dit le nombre de colonies infestées depuis $2,3, \theta_{\mathrm{i}}$ années à la date $t+1$ est respectivement le nombre de colonies infestées depuis 1, 2, $\theta_{i-1}$ années à la date $t$.

On en déduit alors le vecteur des susceptibles, par différence entre le nombre de colonies initiales et le nombre de colonies infestées :

$$
S_{i}(t+1)=S_{i}(1)-\sum_{j=1}^{\theta_{i}} J_{i, t+1, j}
$$

\section{Annexe 2 \\ Valeur des différents paramètres utilisés (sauf mention explicite dans le texte)}

Horizon temporel : 100 ans

Taux d'actualisation marginal : $4 \%$ jusqu'à 30 ans, $2 \%$ au-delà. Masse de miel produite par colonie et par an, sans pathologie : $15 \mathrm{~kg}$.

Prix moyen du kg de miel en gros, y compris les produits dérivés commercialisés : $6 € / \mathrm{kg}$.

Produits divers et valeur existentielle d'une colonie par an (vente de cire, d'essaims, valeur d'existence etc.) : $10 € /$ colonie.

Valeur en euros de la pollinisation pour une colonie : $5 € / \mathrm{an}$. 
Montant forfaitaire de la taxe MSA pour un rucher de 50 colonies, la première année : $550 €$.

Montant du taux de cotisation apicole MSA les années suivantes entre 50 et 200 ruches : 0,24.

Impôt sur le revenu, pour ruchers supérieurs à 10 colonies : $2 € /$ an et par colonie ( $20 \%$ d'un revenu forfaitaire de $10 € /$ colonie). Coût de traitement et de remplacement d'une colonie atteinte par la pathologie, une fois détectée : $100 €$.

Taux de production de miel, la première année après le remplacement d'une colonie : $50 \%$.

L'État (au sens large) retire indirectement des utilités des apiculteurs $50 \%$ d'utilité complémentaire par l'effet filière et les activités indirectes, en plus de la valeur de la pollinisation, de l'impôt sur le revenu et des taxes directes ou indirectes, dont la MSA.

Matrice $K$ de diffusion ou de propagation de la pathologie (indice 1 : grand rucher ; 2 : moyen rucher ; 3 : petit rucher):

$$
K=0,0025 *\left(\begin{array}{ccc}
1 & 1 & 1 \\
1 & 1 & 1 \\
1 & 1 & 1
\end{array}\right)
$$

Conditions initiales et paramètres de retard :

$$
\begin{gathered}
J_{1,1,1}=0,000834 ; J_{1,1,2}=0 ; J_{1,1,3}=0 \\
S(1)=\left(\begin{array}{r}
471,75 \\
69,59 \\
4,54
\end{array}\right) ; \theta=\left(\begin{array}{l}
1 \\
3 \\
5
\end{array}\right)
\end{gathered}
$$

Nombre de ruchers dans chaque catégorie :

$$
N=\left(\begin{array}{c}
63 \\
245 \\
1199
\end{array}\right)
$$




\section{Annexe 3 \\ Répartition du coût au prorata des ruchers (RUC)}

Rappelons que le nombre de ruchers est de 1507 avec une répartition de $4,18 \%$ de grands, $16,26 \%$ de moyens et $79,56 \%$ de petits.

Le coût annuel de la mesure étant toujours noté $C$, l'analyse coût-bénéfice (ACB) pour les grands ruchers sera positive si et seulement si $27,2 \mathrm{k} €-0,0418 C>0$, soit $C<650,7 \mathrm{k} €$. Pour les moyens l'ACB est positive si et seulement si $C<558,9 \mathrm{k} €$ et pour les petits $C<6,30 \mathrm{k} €$. Les petits apiculteurs sont peu bénéficiaires de la mesure et une grande part du coût repose sur eux, de ce fait cette mesure n'est acceptable pour eux que si elle est peu onéreuse.

En conséquence, si $C<6,30 \mathrm{k} €$, tous sont bénéficiaires et il y a unanimité pour mettre en ouvre la mesure, si ce sont les apiculteurs qui décident. Ils sont en cela appuyés par l'État dont l'ACB de la mesure est, elle aussi, positive, à hauteur de $37,5 \mathrm{k} €$ qui peut prendre totalement ou partiellement à sa charge le coût de la mesure. Cet intervalle est ainsi extensible jusqu'à $C<43,8 \mathrm{k} €$.

Si $6,30<C<558,9 \mathrm{k} €$, seuls les moyens et les grands apiculteurs sont bénéficiaires, ainsi qu'éventuellement l'État. La mesure sera adoptée si le mode de décision est $B, C, E, F, H$ ou $K$. Dans ce cas les petits apiculteurs sont perdants. L'État peut participer au financement de la mesure, au maximum à hauteur de $37,5 \mathrm{k} €$, ce qui permet d'étendre l'intervalle jusqu'à la valeur maximale de 596,4 k€. Dans certains cas que nous ne détaillons pas ici, cette participation de l'État peut être utilisée de manière à ce que les petits apiculteurs ne soient pas perdants.

Si 558,9 $<C<650,7 \mathrm{k} €$, seuls les grands apiculteurs sont bénéficiaires, et éventuellement l'État. La mesure sera adoptée si le mode de décision est $B, C$ ou $H$. Les petits et moyens apiculteurs sont perdants. Comme précédemment, l'État peut étendre cet intervalle tant que $C<650,7+37,5=688,2 \mathrm{k} €$. 
194 Jean-Philippe Terreaux

Si $650,7<C<688,2 \mathrm{k} €$, l'intervention de l'État est nécessaire pour l'adoption de la mesure, qui ne sera adoptée que si le mode de décision permet aux seuls grands apiculteurs d'emporter la décision, comme dans le cas précédent.

Enfin, si $688,2 \mathrm{k} €<C$, la mesure est trop onéreuse. 


\section{Notes}

1. Colonie : ensemble de la reine et des différents types d'abeilles occupant une même ruche. Ruche : lieu d'hébergement. Rucher : ensemble de ruches ou ensemble de colonies appartenant à un même apiculteur. Un apiculteur peut posséder plusieurs ruchers.

2. Dans sa structure à cinq départements : Dordogne, Gironde, Landes, Lot-etGaronne et Pyrénées-Atlantiques.
3. Valeur totale divisée par la somme des coefficients d'actualisation sur la période considérée.

4. À cette date, le modèle donne un nombre de colonies atteintes de 186 chez les petits apiculteurs, $522 \mathrm{chez}$ les moyens et 645 chez les grands. 


\section{Références}

L.J.S. Allen et A.M. Burgin [2000] : Comparison of Deterministic and Stochastic SIS and SIR Models in Discrete Time, Mathematical Biosciences, 163, pp. 1-33.

K. J. Arrow [1963] : Social Choice and Individual Values, ${ }^{\text {ème }}$ éd., John Wiley et Sons, New York, 124 p.

S. Baliga et E. Maskin [2003] : Mechanism Design for the Environment, in Handbook of Environmental Economics, vol. 1, K.G. Mäler et J.R. Vincent éd., Elsevier Science B.V., pp. 305-324.

F. Ball et P. Neal [2002] : A General Model for Stochastic SIR Epidemics with two Levels of Mixing, Mathematical Biosciences, 180, pp. 73-102.

CGP - Commissariat général au plan [2005] : Révision du taux d'actualisation des investissements publics, L. Baumstark (rapporteur), P. Hirtzman (coordinateur), rapport du CGP, Paris, 112 p.

M.P. Chauzat, M. Laurent, M.P. Rivière, C. Saugeon, P. Hendrikx et M. RibiereChabert [2014] : Epilobee, a Pan-european Study on Honeybee Colony Losses 20122013, mimeo, 32 p.

S. Datta, J.C. Bull, G.E. Budge et M.J. Keeling [2015] : Modelling the Spread of American Foulbrood in Honeybees, Journal of the Royal Society Interface, 10, 20130650, $16 \mathrm{p}$.

FAO [2006]: Honey Bee Diseases and Pests : A Practical Guide, 34 p.

I. Fries et S. Camazine [2001] : Implications of Horizontal and Vertical Pathogen Transmission for Honey Bee Epidemiology, Apidologie, 32, pp. 199-214.
N. Gallai, J.M. Salles, J. Settele et B.E. Vaissière [2009] : Economic Valuation of the Vulnerability of World Agriculture Confronted with Pollinator Decline, Ecological Economics, pp. 810-821.

E. Genersch [2010] : American Foulbrood in Honeybees and its Causative Agent, Paenibacillus Larvae, Journal of Invertebrate Pathology, 103, pp. S10-S19.

E. Genersch, J.D. Evans et I. Fries [2010] : Honey Bee Disease Overview - Preface, Journal of Invertebrate Pathology, 103, pp. S2-S4.

F. Gerster [2012] : Plan de développement durable de l'apiculture, ministère chargé de l'agriculture, CGAAER n ${ }^{\circ} 11$ 174-01, $31 \mathrm{p}$.

J.R. Green et J.J. Laffont [1986] : Partially Verifiable Information and Mechanism Design, Review of Economic Studies, 53, pp. 447-456.

H.W. Hethcote [1989] : Three Basic Epidemiological Models, in S.A. Levin, T.G. Hallam, L.J. Gross éd. Applied Mathematical Ecology, Biomathematics, vol. 18, Springer-Verlag, Berlin, pp. 119-144.

A. Kakhbod [2013] : Resource Allocation in Decentralized Systems with Strategic Agents - An Implementation Theory Approach, Springer, $88 \mathrm{p}$.

F.H. Knight [1947] : Human Nature and World Democracy, American Journal of Sociology, 49 (5), pp. 408-420. 
J.J. Laffont et D. Martimort [2002] : The Theory of Incentives - the Principal Agent Model, Princeton University Press, $421 \mathrm{p}$.

M.O. Lorenz [1905] : Methods of Measuring the Concentration of Wealth, Publications of the American Statistical Association, 9 (70), pp. 209-219.

A.C. Mill, S.P. Rushton, M.D.F. Shirley, G.C. Smith, P. Mason, M.A. Brown et G.E. Budge [2014] : Clustering, Persistence and Control of a Pollinator Brood Disease: Epidemiology of American Foulbrood, Environmental Microbiology, 16 (12), pp. 3753-3763.

J.D. Murray [2002] : Mathematical Biology, vol. I: An Introduction, Springer, $551 \mathrm{p}$.

J. Priestley [1768] : An Essay on the First Principles of Government, $2^{\text {ème }}$ éd. J. Johnson éd., Londres, 317 p.
A. Rapaport, S. Sraidi et J.P. Terreaux [2003] : Optimality of Greedy and Sustainable Policies in the Management of Renewable Resources, Optimal Control Applications and Methods, 24 (1), pp. 23-44.

J.P. Terreaux [2008] : Taux d'actualisation décroissants et cohérence temporelle des décisions de sylviculture, Revue forestière française, 60 (4), pp. 467-476.

J.P. Terreaux [2014] : Nouveaux espaces d'échanges et nouvelles frontières de la démocratie, Cités, 60, pp. 177-186.

Y.C. Zarka [2010] : Pourquoi repenser la démocratie ? in Y.C. Zarka éd. Repenser la démocratie, Armand Colin, Paris, $615 \mathrm{p}$. 